\title{
A Metric Version of Poincaré's Theorem Concerning Biholomorphic Inequivalence of Domains
}

\section{Bas Lemmens ${ }^{1}$}

Received: 7 September 2020 / Accepted: 9 February 2022 / Published online: 7 March 2022

(c) The Author(s) 2022

\begin{abstract}
We show that if $Y_{j} \subset \mathbb{C}^{n_{j}}$ is a bounded strongly convex domain with $C^{3}$-boundary for $j=1, \ldots, q$, and $X_{j} \subset \mathbb{C}^{m_{j}}$ is a bounded convex domain for $j=1, \ldots, p$, then the product domain $\prod_{j=1}^{p} X_{j} \subset \mathbb{C}^{m}$ cannot be isometrically embedded into $\prod_{j=1}^{q} Y_{j} \subset \mathbb{C}^{n}$ under the Kobayashi distance, if $p>q$. This result generalises Poincaré's theorem which says that there is no biholomorphic map from the polydisc onto the Euclidean ball in $\mathbb{C}^{n}$ for $n \geq 2$. The method of proof only relies on the metric geometry of the spaces and will be derived from a more general result for products of proper geodesic metric spaces with the sup-metric. In fact, the main goal of the paper is to establish a general criterion, in terms of certain asymptotic geometric properties of the individual metric spaces, that yields an obstruction for the existence of an isometric embedding between product metric spaces.
\end{abstract}

Keywords Product metric spaces · Product domains - Kobayashi distance - Isometric embeddings $\cdot$ Metric compactification $\cdot$ Busemann points $\cdot$ Detour distance

Mathematics Subject Classification Primary 32F45 - Secondary 51 F99

\section{Introduction}

Numerous theorems in several complex variables are instances of results in metric geometry. In this paper, we shall see that a classic theorem due to Poincaré [22], which says that there is no biholomorphic map from the polydisc $\Delta^{n}$ onto the (open) Euclidean ball $B_{n}$ in $\mathbb{C}^{n}$ if $n \geq 2$, is a case in point. In fact, it is known $[19,29,30]$ that there exists no surjective Kobayashi distance isometry of $\Delta^{n}$ onto $B_{n}$ if $n \geq 2$. More

The author gratefully acknowledges the support of the EPSRC (Grant EP/R044228/1).

$凶 \quad$ Bas Lemmens

B.Lemmens@kent.ac.uk

1 School of Mathematics, Statistics \& Actuarial Science, University of Kent, Canterbury CT2 7NX, UK 
generally, one may wonder when it is possible to isometrically embed a product domain $\prod_{j=1}^{p} X_{j} \subset \mathbb{C}^{m}$ into another product domain $\prod_{j=1}^{q} Y_{j} \subset \mathbb{C}^{n}$ under the Kobayashi distance. In this paper, we show, among other results, the following theorem.

Theorem 1.1 Suppose that $X_{j} \subset \mathbb{C}^{m_{j}}$ is a bounded convex domain for $j=1, \ldots, p$, and $Y_{j} \subset \mathbb{C}^{n_{j}}$ is a bounded strongly convex domain with $C^{3}$-boundary for $j=$ $1, \ldots, q .$. If $p>q$, then there is no isometric embedding of $\prod_{j=1}^{p} X_{j}$ into $\prod_{j=1}^{q} Y_{j}$ under the Kobayashi distance.

Note that Poincaré's theorem is a special case where $p=n \geq 2$ and $q=1$, as the boundary of the Euclidean ball is smooth. The case where $\sum_{j} m_{j}=\sum_{j} n_{j}$ and the isometry is surjective was analysed by Zwonek [29, Theorem 2.2.5] who used different methods.

A key property of the Kobayashi distance is the product property, see [13, Theorem 3.1.9]. Indeed, if $X_{j} \subset \mathbb{C}^{m_{j}}$ is a bounded convex domain for $j=1, \ldots, p$, then the Kobayashi distance, $k_{X}$, on the product domain $X:=\prod_{j=1}^{p} X_{j}$ satisfies

$k_{X}(w, z)=\max _{j=1, \ldots, p} k_{X_{j}}\left(w_{j}, z_{j}\right)$ for all $w=\left(w_{1}, \ldots, w_{p}\right), z=\left(z_{1}, \ldots, z_{p}\right) \in X$.

In view of the product property, it is natural to consider product metric spaces with the sup-metric. Given metric spaces $\left(M_{j}, d_{j}\right), j=1, \ldots, p$, the product metric space $\left(\prod_{j=1}^{p} M_{j}, d_{\infty}\right)$ is given by

$$
d_{\infty}(x, y):=\max _{j} d_{j}\left(x_{j}, y_{j}\right) \text { for } x=\left(x_{1}, \ldots, x_{p}\right), y=\left(y_{1}, \ldots, y_{p}\right) \in \prod_{j=1}^{p} M_{j} .
$$

In this general context, it is interesting to understand when one can isometrically embed a product metric space into another one. The main goal of this paper is to establish a general criterion, in terms of certain asymptotic geometric properties of the individual metric spaces, that yields an obstruction for the existence of an isometric embedding between product metric spaces, and to show how this criterion can be used to derive Theorem 1.1.

The key concepts from metric geometry involved are as follows: the horofunction boundary of proper geodesic metric spaces, almost geodesics, Busemann points, the detour distance, and the parts of the horofunction boundary, which will all be recalled in the next section. Our main result is the following.

Theorem 1.2 Suppose that $\left(M_{j}, d_{j}\right)$ is a proper geodesic metric space containing an almost geodesic sequence for $j=1, \ldots, p$, and $\left(N_{j}, \rho_{j}\right)$ is a proper geodesic metric space such that all its horofunctions are Busemann points, and $\delta\left(h_{j}, h_{j}^{\prime}\right)=\infty$ for all $h_{j} \neq h_{j}^{\prime}$ Busemann points of $\left(N_{j}, \rho_{j}\right)$, for $j=1, \ldots, q$. If $p>q$, then there exists no isometric embedding of $\left(\prod_{j=1}^{p} M_{j}, d_{\infty}\right)$ into $\left(\prod_{j=1}^{q} N_{j}, d_{\infty}\right)$.

The assumption that each horofunction is a Busemann point and that any two distinct Busemann points lie at infinite detour distance from each other is a type of regularity condition on the asymptotic geometry of the space, which is satisfied by numerous 
metric spaces, such as finite dimensional normed spaces with smooth norms [24], Hilbert geometries on bounded strictly convex domains with $C^{1}$-boundary [25], and, as we shall see in Lemma 3.3, Kobayashi metric spaces $\left(D, k_{D}\right)$, where $D \subset \mathbb{C}^{n}$ is a bounded strongly convex domain with $C^{3}$-boundary.

It turns out that the parts of the horofunction boundary and the detour distance in product metric spaces have a special structure that is closely linked to a quotient space of $\left(\mathbb{R}^{n}, 2\|\cdot\|_{\infty}\right)$, where $\|x\|_{\infty}=\max _{j}\left|x_{j}\right|$. More precisely, if we let $\operatorname{Sp}(\mathbf{1}):=$ $\left\{\lambda(1, \ldots, 1) \in \mathbb{R}^{n}: \lambda \in \mathbb{R}\right\}$, then the quotient space $\mathbb{R}^{n} / \operatorname{Sp}(\mathbf{1})$ with respect to $2\|\cdot\|_{\infty}$ has the variation norm as the quotient norm, which is given by

$$
\|\bar{x}\|_{\mathrm{var}}:=\max _{j} x_{j}+\max _{j}\left(-x_{j}\right) \text { for } \bar{x} \in \mathbb{R}^{n} / \operatorname{Sp}(\mathbf{1}),
$$

see [17, Sect. 4]. It is known, e.g. [14, Proposition 2.2.4], that $\left(\mathbb{R}^{n} / \operatorname{Sp}(\mathbf{1}),\|\cdot\|_{\text {var }}\right)$ is isometric to the Hilbert metric space on the open $(n-1)$-dimensional simplex.

We show in Theorem 2.8 that if, for $j=1, \ldots, q$, we have that $\left(N_{j}, \rho_{j}\right)$ is a proper geodesic metric space such that all its horofunctions are Busemann points, and $\delta\left(h_{j}, h_{j}^{\prime}\right)=\infty$ for all $h_{j} \neq h_{j}^{\prime}$ Busemann points of $\left(N_{j}, \rho_{j}\right)$, then each part of $\left(\prod_{j=1}^{q} N_{j}, d_{\infty}\right)$ is isometric to $\left(\mathbb{R}^{n} / \operatorname{Sp}(\mathbf{1}),\|\cdot\|_{\text {var }}\right)$ for some $1 \leq n \leq q$.

The horofunctions for the product of two metric spaces have been considered by Walsh [26, Sect. 8]. Some of our results are extensions of his work to arbitrary finite products, and the ideas of some of the proofs are quite similar. For the reader's convenience, we give full proofs and provide comments on the relation with the work in [26] where relevant.

The work in this paper has links to work by Bracci and Gaussier [7] who studied the interaction between topological properties and the metric geometry of hyperbolic complex spaces. It is also worth mentioning that various other aspects of the metric geometry of product metric spaces have been studied in context of Teichmüller space in $[9,20]$.

\section{The Metric Compactification of Product Spaces}

In our set-up, we will follow the terminology in [12], which contains further references and background on the metric compactification.

Let $(M, d)$ be a metric space, and let $\mathbb{R}^{M}$ be the space of all real functions on $M$ equipped with the topology of pointwise convergence. Fix $b \in M$, which is called the basepoint. Let $\operatorname{Lip}_{b}^{1}(M)$ denote the set of all functions $h \in \mathbb{R}^{M}$ such that $h(b)=0$ and $h$ is 1 -Lipschitz, i.e. $|h(x)-h(y)| \leq d(x, y)$ for all $x, y \in M$. Then $\operatorname{Lip}_{b}^{1}(M)$ is a closed subset of $\mathbb{R}^{M}$. Moreover, as

$$
|h(x)|=|h(x)-h(b)| \leq d(x, b)
$$

for all $h \in \operatorname{Lip}_{b}^{1}(M)$ and $x \in M$, we get that $\operatorname{Lip}_{b}^{1}(M) \subseteq[-d(x, b), d(x, b)]^{M}$, which is compact by Tychonoff's theorem. Thus, $\operatorname{Lip}_{b}^{1}(M)$ is a compact subset of $\mathbb{R}^{M}$. 
Now for $y \in M$ consider the real-valued function

$$
h_{y}(z):=d(z, y)-d(b, y) \text { with } z \in M \text {. }
$$

Then $h_{y}(b)=0$ and $\left|h_{y}(z)-h_{y}(w)\right|=|d(z, y)-d(w, y)| \leq d(z, w)$. Thus, $h_{y} \in \operatorname{Lip}_{b}^{1}(M)$ for all $y \in M$. The closure of $\left\{h_{y}: y \in M\right\}$ is called the metric compactification of $M$ and is denoted $\bar{M}^{h}$. The boundary $\partial \bar{M}^{h}:=\bar{M}^{h} \backslash\left\{h_{y}: y \in M\right\}$ is called the horofunction boundary of $M$, and its elements are called horofunctions. Given a horofunction $h$ and $r \in \mathbb{R}$, the set $\mathcal{H}(h, r):=\{x \in M: h(x)<r\}$ is a called a horoball.

We will assume that the metric space $(M, d)$ is proper, meaning that all closed balls are compact. Such metric spaces are separable, since every compact metric space is separable. It is known that if $(M, d)$ is separable, then the topology of pointwise convergence on $\operatorname{Lip}_{b}^{1}(M)$ is metrizable, and hence each horofunction is the limit of a sequence of functions $\left(h_{y^{n}}\right)$ with $y^{n} \in M$ for all $n \geq 1$. In general, however, horofunctions are limits of nets.

A curve $\gamma: I \rightarrow(M, d)$, where $I$ is a possibly unbounded interval in $\mathbb{R}$, is called a geodesic path if

$$
d(\gamma(s), \gamma(t))=|s-t| \text { for all } s, t \in I
$$

The metric space $(M, d)$ is said to be a geodesic space if for each $x, y \in M$ there exists a geodesic path $\gamma:[a, b] \rightarrow M$ with $\gamma(a)=x$ and $\gamma(b)=y$. A proof of the following well-known fact can be found in [16, Lemma 2.1].

Lemma 2.1 If $(M, d)$ is a proper geodesic metric space, then $h \in \partial \bar{M}^{h}$ if and only if there exists a sequence $\left(y^{n}\right)$ in $M$ such that $h_{y^{n}} \rightarrow h$ and $d\left(y^{n}, b\right) \rightarrow \infty$ as $n \rightarrow \infty$.

It should be noted that in the previous lemma, it is necessary to assume that the metric space is proper. Indeed, consider the star graph with centre vertex $b$ and edges $E_{n}=\left\{b, v^{n}\right\}$ of length $n$ for $n \in \mathbb{N}$. Then the sequence $\left(v^{n}\right)$ in the resulting path metric space, with basepoint $b$, satisfies

$$
\lim _{n \rightarrow \infty} h_{v^{n}}(x)=\lim _{n \rightarrow \infty} d\left(x, v^{n}\right)-d\left(b, v^{n}\right)=d(x, b)=h_{b}(x)
$$

for all $x$, and hence does not yield a horofunction.

A sequence $\left(y^{n}\right)$ in $(M, d)$ is called an almost geodesic sequence if $d\left(y^{n}, y^{0}\right) \rightarrow \infty$ as $n \rightarrow \infty$, and for each $\varepsilon>0$, there exists $N \geq 0$ such that

$$
d\left(y^{m}, y^{k}\right)+d\left(y^{k}, y^{0}\right)-d\left(y^{m}, y^{0}\right)<\varepsilon \text { for all } m \geq k \geq N .
$$

The notion of an almost geodesic sequence goes back to Rieffel [23] and was further developed in $[5,15,24,27]$. In particular, any almost geodesic sequence yields a horofunction, see [23, Lemma 4.5]. 
Lemma 2.2 Let $(M, d)$ be a proper geodesic metric space. If $\left(y^{n}\right)$ is an almost geodesic sequence in $M$, then

$$
h(x)=\lim _{n \rightarrow \infty} d\left(x, y^{n}\right)-d\left(b, y^{n}\right)
$$

exists for all $x \in M$ and, moreover, $h \in \partial \bar{M}^{h}$.

Given a proper geodesic metric space $(M, d)$, a horofunction $h \in \bar{M}^{h}$ is called a Busemann point if there exists an almost geodesic sequence $\left(y^{n}\right)$ in $M$ such that $h(x)=\lim _{n \rightarrow \infty} d\left(x, y^{n}\right)-d\left(b, y^{n}\right)$ for all $x \in M$. We denote the collection of all Busemann points by $\mathcal{B}_{M}$.

It is known that a product metric space $\left(\prod_{j=1}^{p} M_{j}, d_{\infty}\right)$, where

$$
d_{\infty}(x, y)=\max _{j} d_{j}\left(x_{j}, y_{j}\right) \text { for } x=\left(x_{1}, \ldots, x_{p}\right), y=\left(y_{1}, \ldots, y_{p}\right) \in \prod_{j=1}^{p} M_{j}
$$

is a proper geodesic metric space, if each $\left(M_{j}, d_{j}\right)$ is a proper geodesic metric space, see for instance [21, Proposition 2.6.6].

The horofunctions of a product proper geodesic metric spaces have a special form, as the following theorem shows. This theorem is an extension of [26, Proposition 8.1], and the basic idea of the proof is the same.

Theorem 2.3 For $j=1, \ldots$, p let $\left(M_{j}, d_{j}\right)$ be proper geodesic metric spaces. Suppose that $h$ is a horofunction of $\left(\prod_{j=1}^{p} M_{j}, d_{\infty}\right)$ with basepoint $b=\left(b_{1}, \ldots, b_{p}\right)$. If $\left(y^{n}\right)$ is a sequence in $\prod_{j=1}^{p} M_{j}$ converging to $h$, then there exist $J \subseteq\{1, \ldots, p\}$ non-empty, horofunctions $h_{j}$ in $\bar{M}_{j}^{h}$ with basepoint $b_{j}$ for $j \in J, \alpha \in \mathbb{R}^{J}$ with $\min _{j \in J} \alpha_{j}=0$, and a subsequence $\left(y^{n_{k}}\right)$ such that

(1) $d_{\infty}\left(b, y^{n_{k}}\right)-d_{k}\left(b_{j}, y_{j}^{n_{k}}\right) \rightarrow \alpha_{j}$ for all $j \in J$,

(2) $d_{\infty}\left(b, y^{n_{k}}\right)-d_{k}\left(b_{i}, y_{i}^{n_{k}}\right) \rightarrow \infty$ for all $i \notin J$,

(3) $h_{y_{j}^{n_{k}}} \rightarrow h_{j}$ for all $j \in J$.

Moreover, $h$ is of the form,

$$
h(x)=\max _{j \in J} h_{j}\left(x_{j}\right)-\alpha_{j} \text { for } x=\left(x_{1}, \ldots, x_{p}\right) \in \prod_{j=1}^{p} M_{j} .
$$

Proof Let $\left(y^{n}\right)$ be a sequence in $\prod_{j=1}^{p} M_{j}$ such that $\left(h_{y^{n}}\right)$ converges to a horofunction $h$. So $h(x)=\lim _{n \rightarrow \infty} d_{\infty}\left(x, y^{n}\right)-d_{\infty}\left(b, y^{n}\right)$ for all $x \in \prod_{j=1}^{p} M_{j}$. As the product metric space is a proper geodesic metric space, it follows from Lemma 2.1 that $d_{\infty}\left(b, y^{n}\right) \rightarrow \infty$ as $n \rightarrow \infty$. Write $y^{n}:=\left(y_{1}^{n}, \ldots, y_{p}^{n}\right)$ and let $\alpha_{j}^{n}:=d_{\infty}\left(b, y^{n}\right)-d_{j}\left(b_{j}, y_{j}^{n}\right) \geq 0$ for all $j=1, \ldots, p$ and $n \geq 0$.

We may assume, after taking a subsequence, that $h_{y_{j}^{n}}(\cdot):=d_{j}\left(\cdot, y_{j}^{n}\right)-d_{j}\left(b_{j}, y_{j}^{n}\right)$ converges to $h_{j} \in{\overline{M_{j}}}^{h}$ and $\alpha_{j}^{n} \rightarrow \alpha_{j} \in[0, \infty]$ for all $j \in\{1, \ldots, p\}$, and $\alpha_{j_{0}}^{n}=0$ 
for all $n \geq 0$ for some fixed $j_{0} \in\{1, \ldots, p\}$. Let $J:=\left\{j: \alpha_{j}<\infty\right\}$ and note that $j_{0} \in J$. So,

$$
\begin{aligned}
h(x)= & \lim _{n \rightarrow \infty} d_{\infty}\left(x, y^{n}\right)-d_{\infty}\left(b, y^{n}\right)=\lim _{n \rightarrow \infty} \max _{j}\left(d_{j}\left(x_{j}, y_{j}^{n}\right)-d_{j}\left(b_{j}, y_{j}^{n}\right)-\alpha_{j}^{n}\right) \\
& =\max _{j \in J} h_{j}\left(x_{j}\right)-\alpha_{j} .
\end{aligned}
$$

To complete the proof note that $\alpha_{j}<\infty$ implies that $d_{j}\left(b_{j}, y_{j}^{n}\right) \rightarrow \infty$, and hence by Lemma 2.1, we find that $h_{j}$ is a horofunction of $\left(M_{j}, d_{j}\right)$ for $j \in J$.

The following notion will be useful in the sequel. A path $\gamma:[0, \infty) \rightarrow(M, d)$ is called an almost geodesic ray if $d(\gamma(t), \gamma(0)) \rightarrow \infty$, and for each $\varepsilon>0$, there exists $T \geq 0$ such that

$$
d(\gamma(t), \gamma(s))+d(\gamma(s), \gamma(0))-d(\gamma(t), \gamma(0))<\varepsilon \text { for all } t \geq s \geq T
$$

Let $\left(y^{n}\right)$ be an almost geodesic sequence in a geodesic metric space $(M, d)$ and assume that

$$
d\left(y^{n}, y^{0}\right)<d\left(y^{n+1}, y^{0}\right) \text { for all } n \geq 0
$$

For simplicity, we write $\Delta_{n}:=d\left(y^{n}, y^{0}\right)$ and we let $\gamma_{n}:\left[0, d\left(y^{n+1}, y^{n}\right)\right] \rightarrow(M, d)$ be a geodesic path connecting $y^{n}$ and $y^{n+1}$, i.e. $\gamma_{n}(0)=y^{n}$ and $\gamma_{n}\left(d\left(y^{n+1}, y^{n}\right)\right)=$ $y^{n+1}$. for all $n \geq 0$.

We write $I_{n}:=\left[\Delta_{n}, \Delta_{n+1}\right]$ and let $\bar{\gamma}_{n}: I_{n} \rightarrow(M, d)$ be the affine reparametrisation of $\gamma_{n}$ given by

$$
\bar{\gamma}_{n}(t):=\gamma_{n}\left(\frac{d\left(y^{n+1}, y^{n}\right)}{\Delta_{n+1}-\Delta_{n}}\left(t-\Delta_{n}\right)\right) \text { for all } t \in I_{n} .
$$

We call the path $\bar{\gamma}:[0, \infty) \rightarrow(M, d)$ given by

$$
\bar{\gamma}(t):=\bar{\gamma}_{n}(t) \text { for } t \in I_{n}
$$

a ray induced by $\left(y^{n}\right)$. Note that $\bar{\gamma}$ is well defined for all $t \geq 0$ by (2.2).

Lemma 2.4 If $\left(y^{n}\right)$ is an almost geodesic sequence in a geodesic metric space $(M, d)$ converging to a horofunction $h$ and satisfying (2.2), then each ray, $\bar{\gamma}$, induced by $\left(y^{n}\right)$ satisfies:

(i) $\bar{\gamma}$ is an almost geodesic ray and $h_{\bar{\gamma}(t)} \rightarrow h$ as $t \rightarrow \infty$,

(ii) the map $t \mapsto d(\bar{\gamma}(t), \bar{\gamma}(0))$ is continuous on $[0, \infty)$.

Proof We first show that for each $\varepsilon>0$, there exists $T \geq 0$ such that

$$
d\left(\bar{\gamma}(t), y^{n}\right)+d\left(y^{n}, y^{0}\right)-d\left(\bar{\gamma}(t), y^{0}\right)<\varepsilon \text { for all } t \geq T \text { and } n \geq 0 \text { with } t \in I_{n}
$$


To get this inequality, just note that there exists $N \geq 0$ such that

$$
\begin{aligned}
d\left(\bar{\gamma}(t), y^{n}\right)+d\left(y^{n}, y^{0}\right)-d\left(\bar{\gamma}(t), y^{0}\right)= & d\left(y^{n+1}, \bar{\gamma}(t)\right)+d\left(\bar{\gamma}(t), y^{n}\right)+d\left(y^{n}, y^{0}\right) \\
& -d\left(\bar{\gamma}(t), y^{0}\right)-d\left(y^{n+1}, \bar{\gamma}(t)\right) \\
\leq & d\left(y^{n+1}, y^{n}\right)+d\left(y^{n}, y^{0}\right)-d\left(y^{n+1}, y^{0}\right)<\varepsilon,
\end{aligned}
$$

for all $t \in I_{n}$ and $n \geq N$, as $\left(y^{n}\right)$ is an almost geodesic sequence. So to get (2.3), we can take $T=\Delta_{N}$.

To prove that $\bar{\gamma}$ is an almost geodesic ray, we need to show that for each $\varepsilon>0$, there exists $S \geq 0$ such that

$$
d(\bar{\gamma}(t), \bar{\gamma}(s))+d(\bar{\gamma}(s), \bar{\gamma}(0))-d(\bar{\gamma}(t), \bar{\gamma}(0))<\varepsilon \text { for all } t \geq s \geq S
$$

Suppose that $t>s$ are such that $t \in I_{n}$ and $s \in I_{k}$ with $n>k$. Then by using (2.3), we know that for all $n$ and $k$ large,

$$
\begin{aligned}
d(\bar{\gamma}(t), \bar{\gamma}(s))+d(\bar{\gamma}(s), \bar{\gamma}(0))-d(\bar{\gamma}(t), \bar{\gamma}(0)) \leq d(\bar{\gamma}(t), \bar{\gamma}(s))+d\left(\bar{\gamma}(s), y^{k}\right)+d\left(y^{k}, y^{0}\right) \\
\quad-d\left(\bar{\gamma}(t), y^{0}\right) \\
\leq d\left(\bar{\gamma}(t), y^{n}\right)+d\left(y^{n}, \bar{\gamma}(s)\right)+d\left(\bar{\gamma}(s), y^{k}\right) \\
\quad+d\left(y^{k}, y^{0}\right)-d\left(\bar{\gamma}(t), y^{0}\right) \\
<-d\left(y^{n}, y^{0}\right)+d\left(y^{n}, \bar{\gamma}(s)\right)+d\left(\bar{\gamma}(s), y^{k}\right) \\
\quad+d\left(y^{k}, y^{0}\right)+\varepsilon \\
\leq-d\left(y^{n}, y^{0}\right)+d\left(y^{n}, y^{k+1}\right)+d\left(y^{k+1}, \bar{\gamma}(s)\right) \\
\quad+d\left(\bar{\gamma}(s), y^{k}\right)+d\left(y^{k}, y^{0}\right)+\varepsilon \\
=-d\left(y^{n}, y^{0}\right)+d\left(y^{n}, y^{k+1}\right)+d\left(y^{k+1}, y^{k}\right) \\
\quad+d\left(y^{k}, y^{0}\right)+\varepsilon \\
<-d\left(y^{n}, y^{0}\right)+d\left(y^{n}, y^{k+1}\right) \\
\quad+d\left(y^{k+1}, y^{0}\right)+2 \varepsilon<3 \varepsilon .
\end{aligned}
$$

Finally suppose that $t \geq s$ are such that $t, s \in I_{n}$. Then for all $n \geq 0$ large we have that

$$
\begin{aligned}
d(\bar{\gamma}(t), \bar{\gamma}(s))+d(\bar{\gamma}(s), \bar{\gamma}(0))-d(\bar{\gamma}(t), \bar{\gamma}(0))= & d\left(\bar{\gamma}(t), y^{n}\right)-d\left(y^{n}, \bar{\gamma}(s)\right)+d(\bar{\gamma}(s), \bar{\gamma}(0)) \\
& -d(\bar{\gamma}(t), \bar{\gamma}(0)) \\
\leq & d\left(\bar{\gamma}(t), y^{n}\right)+d\left(y^{n}, y^{0}\right)-d\left(\bar{\gamma}(t), y^{0}\right)<\varepsilon .
\end{aligned}
$$

As $\bar{\gamma}$ is an almost geodesic ray, we know by [23, Lemma 4.5] that $h_{\bar{\gamma}}(t) \rightarrow h^{\prime}$, where $h^{\prime}$ is a horofunction. As $\bar{\gamma}\left(\Delta_{n}\right)=y^{n}$ for all $n$, we get that $h^{\prime}=h$.

To prove the second assertion, we note that the affine map

$$
t \mapsto \frac{d\left(y^{n+1}, y^{n}\right)}{\Delta_{n+1}-\Delta_{n}}\left(t-\Delta_{n}\right)
$$


is a continuous map from $I_{n}$ onto $\left[0, d\left(y^{n_{1}}, y^{n}\right)\right]$, and the map $\gamma_{n}:\left[0, d\left(y^{n+1}, y^{n}\right)\right] \rightarrow$ $(M, d)$ is continuous, as $\gamma_{n}$ is a geodesic. Thus, the map $t \mapsto d(\bar{\gamma}(t), \bar{\gamma}(0))$ is continuous on the interior of the interval $I_{n}$ for all $n \geq 0$. To get continuity at the endpoints, we simply note that for all $n \geq 0$,

$$
\lim _{t \rightarrow \Delta_{n}^{-}} d(\bar{\gamma}(t), \bar{\gamma}(0))=d\left(y^{n}, \bar{\gamma}(0)\right)=\lim _{t \rightarrow \Delta_{n}^{+}} d(\bar{\gamma}(t), \bar{\gamma}(0))
$$

which completes the proof.

Lemma 2.5 If $\left(y^{n}\right)$ is an almost geodesic sequence in a geodesic metric space $(M, d)$ satisfying (2.2) and $\bar{\gamma}$ is a ray induced by $\left(y^{n}\right)$, then for each sequence $\left(\beta^{n}\right)$ in $[0, \infty)$ with $\beta^{n+1}>\beta^{n}$ for all $n \geq 0$, there exists sequence $\left(t^{n}\right)$ in $[0, \infty)$ with $t^{n+1}>t^{n}$ for all $n \geq 0$ such that $d\left(\bar{\gamma}\left(t^{n}\right), \bar{\gamma}(0)\right)=\beta^{n}$ for all $n \geq 0$.

Proof Note that as $\bar{\gamma}:[0, \infty) \rightarrow M$ is an almost geodesic by Lemma 2.4(i), we know that $d(\bar{\gamma}(t), \bar{\gamma}(0)) \rightarrow \infty$ as $t \rightarrow \infty$. From Lemma 2.4(ii) we know that $\bar{\gamma}$ is continuous on $[0, \infty)$, so there exists $t_{0}^{n} \geq 0$ such that $d\left(\bar{\gamma}\left(t_{0}^{n}\right), \bar{\gamma}(0)\right)=\beta^{n}$. Now if we let $t^{n}:=\inf \left\{t \geq 0: d(\bar{\gamma}(t), \bar{\gamma}(0))=\beta^{n}\right\}$, then by continuity of $\bar{\gamma}$ we have that $d\left(\bar{\gamma}\left(t^{n}\right), \bar{\gamma}(0)\right)=\beta^{n}$ and $t^{n+1}>t^{n}$ for all $n \geq 0$.

\subsection{Detour Distance}

Suppose that $(M, d)$ is a proper geodesic metric space. Given two Busemann points $h_{1}, h_{2} \in \partial \bar{M}^{h}$, the detour cost is given by

$$
H\left(h_{1}, h_{2}\right):=\inf _{\left(z^{n}\right)} \liminf _{n} d\left(b, z^{n}\right)+h_{2}\left(z^{n}\right),
$$

where the infimum is taken over all sequences $\left(z^{n}\right)$ such that $h_{z^{n}}$ converges to $h_{1}$. It is known, see [15, Lemma 3.1], that if $\left(z^{n}\right)$ is an almost geodesic sequence converging to $h_{1}$ and $\left(w^{m}\right)$ converges to $h_{2}$, then

$H\left(h_{1}, h_{2}\right)=\lim _{n \rightarrow \infty}\left(d\left(b, z^{n}\right)+\lim _{m \rightarrow \infty} d\left(z^{n}, w^{m}\right)-d\left(b, w^{m}\right)\right)=\lim _{n \rightarrow \infty} d\left(b, z^{n}\right)+h_{2}\left(z^{n}\right)$.

The detour distance is given by

$$
\delta\left(h_{1}, h_{2}\right):=H\left(h_{1}, h_{2}\right)+H\left(h_{2}, h_{1}\right) .
$$

Note that for all $m, n \geq 0$, we have that

$$
d\left(b, z^{n}\right)+d\left(z^{n}, w^{m}\right)-d\left(b, w^{m}\right) \geq 0
$$

so that $H\left(h_{1}, h_{2}\right) \geq 0$ for all $h_{1}, h_{2} \in \partial \bar{M}^{h}$. It is, however, possible for $H\left(h_{1}, h_{2}\right)$ to be infinite. It can be shown, see [15, Sect. 3] or [27, Sect. 2], that the detour distance is independent of the basepoint. 
The detour distance was introduced in [5] and has been exploited and further developed in [15, 27]. It is known, see for instance [15, Sect. 3] or [27, Sect. 2], that on $\mathcal{B}_{M} \subseteq \partial \bar{M}^{h}$, the detour distance is symmetric, satisfies the triangle inequality, and $\delta\left(h_{1}, h_{2}\right)=0$ if and only if $h_{1}=h_{2}$. This yields a partition of $\mathcal{B}_{M}$ into equivalence classes, where $h_{1}$ and $h_{2}$ are said to be equivalent if $\delta\left(h_{1}, h_{2}\right)<\infty$. The equivalence class of $h$ will be denoted by $\mathcal{P}(h)$. Thus, the set of Busemann points, $\mathcal{B}_{M}$, is the disjoint union of metric spaces under the detour distance, which are called parts of $\mathcal{B}_{M}$.

Isometric embeddings between proper geodesic metric spaces can be extended to the parts of the metric spaces as detour distance isometries. Indeed, suppose that $\varphi:(M, d) \rightarrow(N, \rho)$ is an isometric embedding, i.e. $\rho(\varphi(x), \varphi(y))=d(x, y)$ for all $x, y \in M$. (Note that $\varphi$ need not be onto.) If $h$ is a Busemann point of $(M, d)$ with basepoint $b$ and $\left(z^{n}\right)$ is an almost geodesic sequence such that $\left(h_{z^{n}}\right)$ converges to $h$, then $\left(u^{n}\right)$, with $u^{n}:=\varphi\left(z^{n}\right)$ for $n \geq 0$, is an almost geodesic sequence in $(N, \rho)$, and hence $\left(h_{u^{n}}\right)$ converges to a Busemann point, say $\varphi(h)$, of $(N, \rho)$ with basepoint $\varphi(b)$.

We note that $\varphi(h)$ is independent of the almost geodesic sequence $\left(z^{n}\right)$. To see this, let $\left(w^{n}\right)$ be another almost geodesic such that $\left(h_{w^{n}}\right)$ converges to $h$. Write $v^{n}:=\varphi\left(w^{n}\right)$ for $n \geq 0$ and let $\varphi(h)^{\prime}$ be the limit of $\left(h_{v^{n}}\right)$. Then

$$
\begin{aligned}
H(h, h) & =\lim _{n \rightarrow \infty} d\left(w^{n}, b\right)+\lim _{m \rightarrow \infty} d\left(w^{n}, z^{m}\right)-d\left(b, z^{m}\right) \\
& =\lim _{n \rightarrow \infty} \rho\left(v^{n}, \varphi(b)\right)+\lim _{m \rightarrow \infty} \rho\left(v^{n}, u^{m}\right)-\rho\left(\varphi(b), u^{m}\right) \\
& =H\left(\varphi(h)^{\prime}, \varphi(h)\right) .
\end{aligned}
$$

Likewise, $H\left(\varphi(h), \varphi(h)^{\prime}\right)=H(h, h)$, and we deduce that $\delta\left(\varphi(h)^{\prime}, \varphi(h)\right)=$ $H\left(\varphi(h)^{\prime}, \varphi(h)\right)+H\left(\varphi(h), \varphi(h)^{\prime}\right)=\delta(h, h)=0$, which shows that $\varphi(h)^{\prime}=\varphi(h)$, as $\varphi(h)^{\prime}$ and $\varphi(h)$ are Busemann points. Thus, there exists a well-defined map $\Phi: \mathcal{B}_{M} \rightarrow \mathcal{B}_{N}$ given by $\Phi(h):=\varphi(h)$.

Lemma 2.6 If $\varphi:(M, d) \rightarrow(N, \rho)$ is an isometric embedding, then $\Phi(\mathcal{P}(h)) \subseteq$ $\mathcal{P}(\varphi(h))$ for all Busemann points $h$ of $(M, d)$ and

$$
\delta\left(h^{\prime}, h\right)=\delta\left(\Phi\left(h^{\prime}\right), \Phi(h)\right) \text { for all } h, h^{\prime} \in \mathcal{B}_{M} .
$$

Proof Let $\left(z^{n}\right)$ and $\left(w^{n}\right)$ be almost geodesic sequences such that $\left(h_{z^{n}}\right)$ converges to $h$ and $\left(h_{w^{n}}\right)$ converges to $h^{\prime}$ in $(M, d)$ with basepoint $b$. Then

$$
\begin{aligned}
H\left(h^{\prime}, h\right) & =\lim _{n \rightarrow \infty} d\left(w^{n}, b\right)+\lim _{m \rightarrow \infty} d\left(w^{n}, z^{m}\right)-d\left(b, z^{m}\right) \\
& =\lim _{n \rightarrow \infty} \rho\left(v^{n}, \varphi(b)\right)+\lim _{m \rightarrow \infty} \rho\left(v^{n}, u^{m}\right)-\rho\left(\varphi(b), u^{m}\right) \\
& =H\left(\varphi(h)^{\prime}, \varphi(h)\right) .
\end{aligned}
$$

Likewise, $H\left(h, h^{\prime}\right)=H\left(\varphi(h), \varphi(h)^{\prime}\right)$, so that $\delta\left(h^{\prime}, h\right)=\delta\left(\Phi\left(h^{\prime}\right), \Phi(h)\right)$, which completes the proof.

It could happen that all parts consist of a single Busemann point, but there are also natural instances where there are nontrivial parts. In case of products of metric spaces 
coming from proper geodesic metric spaces, it turns out that the parts and the detour distance have a special structure that is linked to the quotient space, $\left(\mathbb{R}^{n} / \operatorname{Sp}(\mathbf{1}),\|\cdot\|_{\text {var }}\right)$ given in (1.1), as shown by the following proposition.

Proposition 2.7 If, for $j=1, \ldots, p,\left(M_{j}, d_{j}\right)$ is a proper geodesic metric space with almost geodesic sequence $\left(y_{j}^{n}\right)$ and corresponding Busemann point $h_{j}$ with basepoint $y_{j}^{0}$, and $J \subseteq\{1, \ldots, p\}$ is non-empty, then the following assertions hold:

(i) For $\alpha \in \mathbb{R}^{J}$ with $\min _{j \in J} \alpha_{j}=0$ the function $h:\left(\prod_{j=1}^{p} M_{j}, d_{\infty}\right) \rightarrow \mathbb{R}$ given by,

$$
h(x)=\max _{j \in J} h_{j}\left(x_{j}\right)-\alpha_{j}, \text { for } x \in \prod_{j=1}^{p} M_{j},
$$

is a Busemann point with basepoint $y^{0}=\left(y_{1}^{0}, \ldots, y_{p}^{0}\right)$. Moreover, there exists an almost geodesic sequence $\left(z^{n}\right)$ converging to $h$, where $\left(z_{j}^{n}\right)$ is an almost geodesic converging to $h_{j}$ for $j \in J$ such that for all $n \geq 1$, we have that $d_{\infty}\left(z^{n}, y^{0}\right)-$ $d_{j}\left(z_{j}^{n}, y_{j}^{0}\right)=\alpha_{j}$ for $j \in J$, and $d_{i}\left(z_{i}^{n}, y_{i}^{0}\right)=0$ for all $i \notin J$.

(ii) If $\beta \in \mathbb{R}^{J}$ with $\min _{j \in J} \beta_{j}=0$ and $h^{\prime}$ is a Busemann point with basepoint $y^{0}$ of the form,

$$
h^{\prime}(x)=\max _{j \in J} h_{j}\left(x_{j}\right)-\beta_{j}, \text { for } x \in \prod_{j=1}^{p} M_{j},
$$

then $\delta\left(h, h^{\prime}\right)=\|\alpha-\beta\|_{\text {var. }}$.

(iii) For $h$ as in (2.5) the part $(\mathcal{P}(h), \delta)$ contains an isometric copy of $\left(\mathbb{R}^{J} / \mathrm{Sp}(\mathbf{1}), \|\right.$.

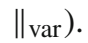

Proof We know there exists an almost geodesic sequence $\left(y_{j}^{n}\right)$ in $\left(M_{j}, d_{j}\right)$ such that $h_{y_{j}^{n}} \rightarrow h_{j}$ for each $j \in J$. As $d_{j}\left(y_{j}^{n}, b_{j}\right) \rightarrow \infty$, we can take a subsequence and assume that $d_{j}\left(y_{j}^{n+1}, y_{j}^{0}\right)>d_{j}\left(y_{j}^{n}, y_{j}^{0}\right)>\alpha_{j}$ for all $n \geq 1$. Let $\bar{\gamma}_{j}$ be a ray induced by $\left(y_{j}^{n}\right)$.

For $j \in J$ we get from Lemma 2.5 a sequence $\left(t_{j}^{n}\right)$ in $[0, \infty)$ with $t_{j}^{0}=0$ and

$$
d_{j}\left(\bar{\gamma}_{j}\left(t_{j}^{n}\right), y_{j}^{0}\right)=\left(\max _{i \in J} d_{i}\left(y_{i}^{n}, y_{i}^{0}\right)\right)-\alpha_{j} \geq 0 \text { for all } n \geq 1
$$

Let $z^{0}:=\left(y_{1}^{0}, \ldots, y_{p}^{0}\right)$ and for $n \geq 1$ define $z^{n}=\left(z_{1}^{n}, \ldots, z_{p}^{n}\right) \in \prod_{j=1}^{p} M_{j}$ by $z_{j}^{n}:=\bar{\gamma}_{j}\left(t_{j}^{n}\right)$ if $j \in J$, and $z_{j}^{n}:=y_{j}^{0}$ otherwise.

As $\min _{j \in J} \alpha_{j}=0$, we get by construction that

$$
d_{\infty}\left(z^{n}, z^{0}\right)=\max _{i \in J} d_{i}\left(y_{i}^{n}, y_{i}^{0}\right)=d_{j}\left(z_{j}^{n}, z_{j}^{0}\right)+\alpha_{j} \text { for all } n \geq 1 \text { and } j \in J .
$$

Moreover, it follows from Lemma 2.4 that $\left(z_{j}^{n}\right)$ is an almost geodesic converging to $h_{j}$ for $j \in J$. 
We claim that $\left(z^{n}\right)$ is an almost geodesic sequence in $\left(\prod_{j=1}^{p} M_{j}, d_{\infty}\right)$. Indeed, note that for $n \geq k \geq 0$, we have that

$$
d_{\infty}\left(z^{n}, z^{k}\right)+d_{\infty}\left(z^{k}, z^{0}\right)-d_{\infty}\left(z^{n}, z^{0}\right)=d_{j}\left(z_{j}^{n}, z_{j}^{k}\right)+d_{\infty}\left(z^{k}, z^{0}\right)-d_{\infty}\left(z^{n}, z^{0}\right)
$$

for some $j=j(n, k) \in J$, as $d_{j}\left(z_{j}^{n}, z_{j}^{k}\right)=0$ for all $j \notin J$. As $J$ is non-empty, we find for all $n \geq k$ large that

$$
d_{\infty}\left(z^{n}, z^{k}\right)+d_{\infty}\left(z^{k}, z^{0}\right)-d_{\infty}\left(z^{n}, z^{0}\right)=d_{j}\left(z_{j}^{n}, z_{j}^{k}\right)+d_{j}\left(z_{j}^{k}, z_{j}^{0}\right)+\alpha_{j}-d_{j}\left(z_{j}^{n}, z_{j}^{0}\right)-\alpha_{j}<\varepsilon .
$$

Also for $n \geq 0$ large and $x \in \prod_{j=1}^{p} M_{j}$, we have that

$$
h_{z^{n}}(x)=\max _{j \in J}\left(d_{j}\left(x_{j}, z_{j}^{n}\right)-d_{\infty}\left(z^{n}, z^{0}\right)\right)=\max _{j \in J}\left(d_{j}\left(x_{j}, z_{j}^{n}\right)-d_{j}\left(z_{j}^{n}, z_{j}^{0}\right)-\alpha_{j}\right) .
$$

Letting $n \rightarrow \infty$ gives

$$
h(x)=\max _{j \in J} h_{j}\left(x_{j}\right)-\alpha_{j} \text { for all } x \in \prod_{j=1}^{p} M_{j}
$$

and shows that $h$ is a Busemann point with basepoint $y^{0}=\left(y_{1}^{0}, \ldots, y_{p}^{0}\right)$. This completes the proof of assertion (i).

To prove the second assertion, we know from the first assertion that there exists an almost geodesic sequence $\left(w^{n}\right)$ converging to $h^{\prime}$, where $\left(w_{j}^{n}\right)$ is an almost geodesic converging to $h_{j}$ and $d_{\infty}\left(w^{n}, y^{0}\right)-d_{j}\left(w_{j}^{n}, y_{j}^{0}\right)=\beta_{j}$ for $j \in J$. So, we get that

$$
\begin{aligned}
\max _{j \in J}\left(\beta_{j}-\alpha_{j}\right) & =\max _{j \in J}\left(H\left(h_{j}, h_{j}\right)+\beta_{j}-\alpha_{j}\right) \\
& =\max _{j \in J}\left(\lim _{n \rightarrow \infty} d_{j}\left(w_{j}^{n}, y_{j}^{0}\right)+\beta_{j}+h_{j}\left(w_{j}^{n}\right)-\alpha_{j}\right) \\
& =\max _{j \in J}\left(\lim _{n \rightarrow \infty} d_{\infty}\left(w^{n}, y^{0}\right)+h_{j}\left(w_{j}^{n}\right)-\alpha_{j}\right) \\
& =\lim _{n \rightarrow \infty} \max _{j \in J}\left(d_{\infty}\left(w^{n}, y^{0}\right)+h_{j}\left(w_{j}^{n}\right)-\alpha_{j}\right) \\
& =\lim _{n \rightarrow \infty} d_{\infty}\left(w^{n}, y^{0}\right)+h\left(w^{n}\right) .
\end{aligned}
$$

Interchanging the roles of $h$ and $h^{\prime}$, we find that

$$
\delta\left(h^{\prime}, h\right)=H\left(h^{\prime}, h\right)+H\left(h, h^{\prime}\right)=\max _{j \in J}\left(\beta_{j}-\alpha_{j}\right)+\max _{j \in J}\left(\alpha_{j}-\beta_{j}\right)=\|\alpha-\beta\|_{\mathrm{var}} .
$$

The final assertion is a direct consequence of the previous two, as $\left(S,\|\cdot\|_{\text {var }}\right)$ with $S:=\left\{\alpha \in \mathbb{R}^{J}: \min _{j \in J} \alpha_{j}=0\right\}$ is isometric to $\left(\mathbb{R}^{J} / \operatorname{Sp}(\mathbf{1}),\|\cdot\|_{\text {var }}\right)$. 
Proposition 2.7 is related to [26, Propositions 8.3 and 8.4], where the Busemann points for the product of two metric spaces are characterised and the detour cost is determined.

It is interesting to understand when a part $(\mathcal{P}(h), \delta)$ is isometric to $\left(\mathbb{R}^{J} / \operatorname{Sp}(\mathbf{1}), \|\right.$. $\left.\|_{\text {var }}\right)$.

Theorem 2.8 If, for $j=1, \ldots, q,\left(N_{j}, \rho_{j}\right)$ is a proper geodesic metric space such that all horofunctions are Busemann points, and $\delta\left(h_{j}, h_{j}^{\prime}\right)=\infty$ for all $h_{j} \neq h_{j}^{\prime}$ Busemann points of $\left(N_{j}, \rho_{j}\right)$, then every horofunction $h$ of $\left(\prod_{j=1}^{q} N_{j}, d_{\infty}\right)$ is a Busemann point, and $(\mathcal{P}(h), \delta)$ is isometric to $\left(\mathbb{R}^{J} / \operatorname{Sp}(\mathbf{1}),\|\cdot\|_{\text {var }}\right)$ for some $J \subseteq\{1, \ldots, q\}$.

Proof Let $h$ be a horofunction of $\left(\prod_{j=1}^{q} N_{j}, d_{\infty}\right)$ with respect to basepoint $b=$ $\left(b_{1}, \ldots, b_{q}\right)$. By Theorem 2.3 we know that $h$ is of the form

$$
h(x)=\max _{j \in J} h_{j}\left(x_{j}\right)-\alpha_{j} \text { for } x \in \prod_{j=1}^{q} N_{j},
$$

and $h_{j}$ is a horofunction of $\left(N_{j}, \rho_{j}\right)$ with respect to basepoint $b_{j}$ for each $j \in J$. As each horofunction of $\left(N_{j}, \rho_{j}\right)$, is a Busemann point, there exists an almost geodesic sequence $\left(y_{j}^{n}\right)$ such that $\left(h_{y_{j}^{n}}\right)$ converges to $h_{j}$ with basepoint $b_{j}$.

For $j \notin J$ let $y_{j}^{0}=b_{j}$ and define $y^{0}:=\left(y_{1}^{0}, \ldots, y_{q}^{0}\right)$. Let $h_{j}^{*}$ be the Busemann point obtained by changing the basepoint of $h_{j}$ to $y_{j}^{0}$, so $h_{j}^{*}\left(x_{j}\right):=h_{j}\left(x_{j}\right)-h_{j}\left(y_{j}^{0}\right)$. Now note that if we change the basepoint for $h$ to $y^{0}$, we get the Busemann point

$$
\begin{aligned}
h^{*}(x) & :=h(x)-h\left(y^{0}\right) \\
& =\max _{j \in J}\left(h_{j}\left(x_{j}\right)-\alpha_{j}\right)-\max _{i \in J}\left(h_{i}\left(y_{i}^{0}\right)-\alpha_{i}\right) \\
& =\max _{j \in J}\left(h_{j}^{*}\left(x_{j}\right)+h_{j}\left(y_{j}^{0}\right)-\alpha_{j}-\max _{i \in J}\left(h_{i}\left(y_{i}^{0}\right)-\alpha_{i}\right)\right) \\
& =\max _{j \in J} h_{j}^{*}\left(x_{j}\right)-\gamma_{j},
\end{aligned}
$$

where $\gamma_{j}:=\max _{i \in J}\left(h_{i}\left(y_{i}^{0}\right)-\alpha_{i}\right)-\left(h_{j}\left(y_{j}^{0}\right)-\alpha_{j}\right) \geq 0$ for $j \in J$ and $\min _{j \in J} \gamma_{j}=0$. It now follows from Proposition 2.7(i) that $h^{*}$ is a Busemann point of $\left(\prod_{j=1}^{q} N_{j}, d_{\infty}\right)$ with respect to basepoint $y^{0}$, and hence $h$ is a Busemann point $\left(\prod_{j=1}^{q} N_{j}, d_{\infty}\right)$ with respect to basepoint $b$. Moreover, there exists an almost geodesic sequence $\left(z^{m}\right)$ converging to $h^{*}$, where $\left(z_{j}^{m}\right)$ is an almost geodesic converging to $h_{j}^{*}$ for $j \in J$, and for all $m \geq 1$ we have that $d_{\infty}\left(z^{m}, y^{0}\right)-d_{j}\left(z_{j}^{m}, y_{j}^{0}\right)=\gamma_{j}$ for $j \in J$, and $d_{i}\left(z_{i}^{m}, y_{i}^{0}\right)=0$ for all $i \notin J$.

To prove the second assertion we note that $(\mathcal{P}(h), \delta)$ is isometric to $\left(\mathcal{P}\left(h^{*}\right), \delta\right)$, since $\delta$ is independent of the basepoint. Let $h^{\prime}$ is a Busemann point of $\left(\prod_{j=1}^{q} N_{j}, d_{\infty}\right)$ with respect to basepoint $y^{0}$ and $\left(w^{n}\right)$ be an almost geodesic converging to $h^{\prime}$. Then 
by Theorem 2.3, we know $h^{\prime}$ is of the form

$$
h^{\prime}(x)=\max _{j \in J^{\prime}} h_{j}^{\prime}\left(x_{j}\right)-\beta_{j}, \text { for } x \in \prod_{j=1}^{q} N_{j},
$$

and, after taking a subsequence, we may assume that $d_{\infty}\left(w_{j}^{n}, y^{0}\right)-d_{k}\left(w_{j}^{n}, y_{j}^{0}\right) \rightarrow \beta_{j}$ for all $j \in J^{\prime}, d_{\infty}\left(w^{n}, y^{0}\right)-d_{i}\left(w_{i}^{n}, y_{i}^{0}\right) \rightarrow \infty$ for all $i \notin J^{\prime}$, and $h_{w_{j}^{n}} \rightarrow h_{j}^{\prime} \in \partial{\overline{N_{j}}}^{h}$ for all $j \in J^{\prime}$.

We claim that if $J \neq J^{\prime}$, or, $J=J^{\prime}$ and $h_{k} \neq h_{k}^{\prime}$ for some $k \in J$, then $\delta\left(h^{*}, h^{\prime}\right)=$ $\infty$. Suppose that $J \neq J^{\prime}$ and $k \in J$, but $k \notin J^{\prime}$. Then

$$
\begin{aligned}
\lim _{m \rightarrow \infty} d_{\infty}\left(w^{n}, z^{m}\right)-d_{\infty}\left(y^{0}, z^{m}\right) & =\lim _{m \rightarrow \infty} d_{\infty}\left(w^{n}, z^{m}\right)-d_{k}\left(y_{k}^{0}, z_{k}^{m}\right)-\gamma_{k} \\
& \geq \lim _{m \rightarrow \infty} d_{k}\left(w_{k}^{n}, z_{k}^{m}\right)-d_{k}\left(y_{k}^{0}, z_{k}^{m}\right)-\gamma_{k} \\
& \geq-d_{k}\left(w_{k}^{n}, y_{k}^{0}\right)-\gamma_{k},
\end{aligned}
$$

so that

$\lim _{n \rightarrow \infty}\left(d_{\infty}\left(w^{n}, y^{0}\right)+\lim _{m \rightarrow \infty} d_{\infty}\left(w^{n}, z^{m}\right)-d_{\infty}\left(y^{0}, z^{m}\right)\right) \geq \lim _{n \rightarrow \infty} d_{\infty}\left(w^{n}, y^{0}\right)-d_{k}\left(w_{k}^{n}, y_{k}^{0}\right)-\gamma_{k}=\infty$.

Thus, $H\left(h^{\prime}, h^{*}\right)=\infty$ and hence $\delta\left(h^{*}, h^{\prime}\right)=\infty$. The case where $k \in J^{\prime}$ and $k \notin J$ can be shown in the same way.

Now suppose that $h_{k}^{*} \neq h_{k}^{\prime}$ for some $k \in J \cap J^{\prime}$. By assumption we know that $\delta\left(h_{k}^{*}, h_{k}^{\prime}\right)=\infty$. Note that

$$
\begin{aligned}
\lim _{n \rightarrow \infty} d_{\infty}\left(w^{n}, y^{0}\right)+h^{*}\left(w^{n}\right) & =\lim _{n \rightarrow \infty} d_{\infty}\left(w^{n}, y^{0}\right)+\max _{j \in J} h_{j}^{*}\left(w_{j}^{n}\right)-\gamma_{j} \\
& \geq \liminf _{n \rightarrow \infty} d_{k}\left(w_{k}^{n}, y_{k}^{0}\right)+h_{k}^{*}\left(w_{k}^{n}\right)-\gamma_{k} .
\end{aligned}
$$

It now follows from (2.4) that $H\left(h^{\prime}, h^{*}\right) \geq H\left(h_{k}^{\prime}, h_{k}^{*}\right)-\gamma_{k}$. Interchanging the roles of $h^{*}$ and $h^{\prime}$, we also get that $H\left(h^{*}, h^{\prime}\right) \geq H\left(h_{k}^{*}, h_{k}^{\prime}\right)-\beta_{k}$, and hence $\delta\left(h^{*}, h^{\prime}\right) \geq$ $\delta\left(h_{k}^{*}, h_{k}^{\prime}\right)-\left(\gamma_{k}+\beta_{k}\right)=\infty$.

On the other hand, if $J=J^{\prime}$ and $h_{j}^{*}=h_{j}^{\prime}$ for all $j \in J$, then it follows from Proposition 2.7(ii) that $\delta\left(h^{*}, h^{\prime}\right)=\|\alpha-\beta\|_{\text {var }}$. Moreover, it follows from that Proposition 2.7(i) that for each $\beta \in \mathbb{R}^{J}$ with $\min _{j \in J} \beta_{j}=0$, there exists a Busemann point in the part of $h^{*}$ of the form (2.6), and hence $\mathcal{P}\left(h^{*}\right)$ consists of all $h^{\prime}$ of the form (2.6), where $\min _{j \in J} \beta_{j}=0$. So if we let $S:=\left\{\beta \in \mathbb{R}^{J}: \min _{j \in J} \beta_{j}=0\right\}$, then $\left(\mathcal{P}\left(h^{*}\right), \delta\right)$ is isometric to $\left(S,\|\cdot\|_{\mathrm{var}}\right)$, which in turn is isometric to the quotient space $\left(\mathbb{R}^{J} / \mathrm{Sp}(\mathbf{1}),\|\cdot\|_{\mathrm{var}}\right)$.

An elementary example is the product space $\left(\mathbb{R}^{n}, d_{\infty}\right)$ where $d_{\infty}(x, y)=$ $\max _{j}\left|x_{j}-y_{j}\right|$. It is easy to verify that $(\mathbb{R},|\cdot|)$ with basepoint 0 has only two horofunctions, namely $h_{+}: x \mapsto x$ and $h_{-}: x \mapsto-x$, both of which are Busemann points 
and $\delta\left(h_{+}, h_{-}\right)=\infty$. So, in this case, we see that the horofunctions $h$ of $\left(\mathbb{R}^{n}, d_{\infty}\right)$ are all Busemann points and of the form,

$$
h(x)=\max _{j \in J} \pm x_{j}-\alpha_{j}
$$

for some $J \subseteq\{1, \ldots, n\}$ non-empty and $\alpha \in \mathbb{R}^{J}$ with $\min _{j \in J} \alpha_{j}=0$, where the sign is fixed for each $j \in J$, see also [10, Theorem 5.2]. Moreover, $(\mathcal{P}(h), \delta)$ is isometric to $\left(\mathbb{R}^{J} / \operatorname{Sp}(\mathbf{1}),\|\cdot\|_{\text {var }}\right)$.

We are now in position to prove Theorem 1.2.

Proof of Theorem 1.2 As each $\left(M_{j}, d_{j}\right)$ contains an almost geodesic sequence $\left(y_{j}^{n}\right)$, it has a Busemann point, say $h_{j}$. We know from Proposition 2.7(i) that the function $h$ of the form, $h(x)=\max _{j=1, \ldots, p} h_{j}\left(x_{j}\right)$, is a Busemann point of $\left(\prod_{j=1}^{p} M_{j}, d_{\infty}\right)$. Moreover, it follows from the third part of the same proposition that $(\mathcal{P}(h), \delta)$ contains an isometric copy of $\left(\mathbb{R}^{p} / \operatorname{Sp}(\mathbf{1}),\|\cdot\|_{\text {var }}\right)$.

Now suppose, for the sake of contradiction, that there exists an isometric embedding $\varphi:\left(\prod_{j=1}^{p} M_{j}, d_{\infty}\right) \rightarrow\left(\prod_{j=1}^{q} N_{j}, d_{\infty}\right)$. Then it follows from Lemma 2.6 that the restriction of $\Phi$ to $\mathcal{P}(h)$ yields an isometric embedding of $(\mathcal{P}(h), \delta)$ into $\left(\mathcal{P}(\Phi(h)), \delta^{\prime}\right)$, where $\delta^{\prime}$ is the detour distance on $\mathcal{P}(\Phi(h))$. It now follows from Theorem 2.8 that $\left(\mathcal{P}(\Phi(h)), \delta^{\prime}\right)$ is isometric to $\left(\mathbb{R}^{n} / \operatorname{Sp}(\mathbf{1}),\|\cdot\|_{\text {var }}\right)$ for some $n \in\{1, \ldots, q\}$. So, $\Phi$ yields an isometric embedding of $\left(\mathbb{R}^{p} / \operatorname{Sp}(\mathbf{1}),\|\cdot\|_{\text {var }}\right)$ into $\left(\mathbb{R}^{n} / \operatorname{Sp}(\mathbf{1}),\|\cdot\|_{\text {var }}\right)$ with $n<p$, which contradicts Brouwer's invariance of domains theorem [8].

\section{Product Domains in $\mathbb{C}^{n}$}

Before we show how we can use Theorem 1.2 to derive Theorem 1.1, we first recall some basic facts concerning the Kobayashi distance, see [13, Chapter 4] for more details. On the disc, $\Delta:=\{z \in \mathbb{C}:|z|<1\}$, the hyperbolic distance is given by

$$
\rho(z, w):=\log \frac{1+\left|\frac{w-z}{1-\bar{z} w}\right|}{1-\left|\frac{w-z}{1-\bar{z} w}\right|}=2 \tanh ^{-1}\left(1-\frac{\left(1-|w|^{2}\right)\left(1-|z|^{2}\right)}{|1-w \bar{z}|^{2}}\right)^{1 / 2} \text { for } z, w \in \Delta .
$$

Given a convex domain $D \subseteq \mathbb{C}^{n}$, the Kobayashi distance is given by

$$
k_{D}(z, w):=\inf \{\rho(\zeta, \eta): \exists f: \Delta \rightarrow D \text { holomorphic with } f(\zeta)=z \text { and } f(\eta)=w\}
$$

for all $z, w \in D$. It was shown by Lempert [18] that on bounded convex domains, the Kobayashi distance coincides with the Caratheodory distance, which is given by

$$
c_{D}(z, w):=\sup _{f} \rho(f(z), f(w)) \text { for all } z, w \in D
$$

where the sup is taken over all holomorphic maps $f: D \rightarrow \Delta$. 
It is known, see [2, Proposition 2.3.10], that if $D \subset \mathbb{C}^{n}$ is bounded convex domain, then $\left(D, k_{D}\right)$ is a proper metric space, whose topology coincides with the usual topology on $\mathbb{C}^{n}$. Moreover, $\left(D, k_{D}\right)$ is a geodesic metric space containing geodesics rays, see [2, Theorem 2.6.19] or [13, Theorem 4.8.6].

In the case of the Euclidean ball $B^{n}:=\left\{\left(z_{1}, \ldots, z_{n}\right) \in \mathbb{C}^{n}:\|z\|^{2}<1\right\}$, where $\|z\|^{2}=\sum_{i}\left|z_{i}\right|^{2}$, the Kobayashi distance has an explicit formula:

$$
k_{B^{n}}(z, w)=2 \tanh ^{-1}\left(1-\frac{\left(1-\|w\|^{2}\right)\left(1-\|z\|^{2}\right)}{|1-\langle z, w\rangle|^{2}}\right)^{1 / 2}
$$

for all $z, w \in B^{n}$, see [2, Chapters 2.2 and 2.3].

On the other hand, on the polydisc $\Delta^{n}:=\left\{\left(z_{1}, \ldots, z_{n}\right) \in \mathbb{C}^{n}: \max _{i}\left|z_{i}\right|<1\right\}$, the Kobayashi distance satisfies

$$
k_{\Delta^{n}}(z, w)=\max _{i} \rho\left(z_{i}, w_{i}\right) \text { for all } w=\left(w_{1}, \ldots, w_{n}\right), z=\left(z_{1}, \ldots, z_{n}\right) \in \Delta^{n}
$$

by the product property, see [13, Theorem 3.1.9].

To determine the horofunctions of $\left(B^{n}, k_{B^{n}}\right)$, with basepoint $b=0$, it suffices to consider limits of sequences $\left(h_{w_{n}}\right)$, where $w_{n} \rightarrow \xi \in \partial B^{n}$ in norm. As

$$
k_{B^{n}}\left(z, w_{n}\right)=\log \frac{\left(\left|1-\left\langle z, w_{n}\right\rangle\right|+\left(\left|1-\left\langle z, w_{n}\right\rangle\right|^{2}-\left(1-\|z\|^{2}\right)\left(1-\left\|w_{n}\right\|^{2}\right)\right)^{1 / 2}\right)^{2}}{\left(1-\|z\|^{2}\right)\left(1-\left\|w_{n}\right\|^{2}\right)},
$$

and

$$
k_{B^{n}}\left(0, w_{n}\right)=\log \frac{\left(1+\left\|w_{n}\right\|\right)^{2}}{1-\left\|w_{n}\right\|^{2}}
$$

it follows that

$$
\begin{aligned}
h(z) & =\lim _{n \rightarrow \infty} k_{B^{n}}\left(z, w_{n}\right)-k_{B^{n}}\left(0, w_{n}\right) \\
& =\log \frac{(|1-\langle z, \xi\rangle|+|1-\langle z, \xi\rangle|)^{2}}{\left(1-\|z\|^{2}\right)(1+\|\xi\|)^{2}} \\
& =\log \frac{|1-\langle z, \xi\rangle|^{2}}{1-\|z\|^{2}} .
\end{aligned}
$$

for all $z \in B^{n}$. Thus, if we write

$$
h_{\xi}(z):=\log \frac{|1-\langle z, \xi\rangle|^{2}}{1-\|z\|^{2}} \text { for all } z \in B^{n}
$$

then we obtain $\partial{\overline{B^{n}}}^{h}=\left\{h_{\xi}: \xi \in \partial B^{n}\right\}$, see also [3, Lemma 2.28] and [11, Remark 3.1]. Moreover, each $h_{\xi}$ is a Busemann point, as it is the limit induced by the geodesic ray $t \mapsto \frac{e^{t}-1}{e^{t}+1} \xi$, for $0 \leq t<\infty$. 
Corollary 3.1 If $h_{\xi}$ and $h_{\eta}$ are distinct horofunctions of $\left(B^{n}, k_{B^{n}}\right)$, then $\delta\left(h_{\xi}, h_{\eta}\right)=$ $\infty$.

Proof If $\xi \neq \eta$ in $\partial B^{n}$, then

$$
\lim _{z \rightarrow \eta} k_{B^{n}}(z, 0)+h_{\xi}(z)=\lim _{z \rightarrow \eta} \log \frac{1+\|z\|}{1-\|z\|}+\log \frac{|1-\langle z, \xi\rangle|^{2}}{1-\|z\|^{2}}=\infty,
$$

which implies that $\delta\left(h_{\xi}, h_{\eta}\right)=\infty$.

Note that if $n=1$, we recover the well-known expression for the horofunctions of the hyperbolic distance on $\Delta$ :

$$
h_{\xi}(z)=\log \frac{|1-z \bar{\xi}|^{2}}{1-|z|^{2}}=\log \frac{|\xi-z|^{2}}{1-|z|^{2}} \text { for all } z \in \Delta
$$

Combining (3.1) with Theorem 2.3 and Proposition 2.7, we get the following.

Corollary 3.2 For $B^{n_{1}} \times \cdots \times B^{n_{q}}$, the Kobayashi distance horofunctions with basepoint $b=0$ are precisely the functions of the form,

$$
h(z)=\max _{j \in J}\left(\log \frac{\left|1-\left\langle z_{j}, \xi_{j}\right\rangle\right|^{2}}{1-\left\|z_{j}\right\|^{2}}-\alpha_{j}\right),
$$

where $J \subseteq\{1, \ldots, q\}$ non-empty, $\xi_{j} \in \partial B^{n_{j}}$ for $j \in J$, and $\min _{j \in J} \alpha_{j}=0$. Moreover, each horofunction is a Busemann point, and $(\mathcal{P}(h), \delta)$ is isometric to $\left(\mathbb{R}^{J} / \operatorname{Sp}(\mathbf{1}),\|\cdot\|_{\text {var }}\right)$.

Corollary 3.2 should be compared with [2, Proposition 2.4.12].

A similar result holds for more general product domains. We know from [2, Theorem 2.6.45] that for each $\xi \in \partial D$, there exists a unique geodesic ray $\gamma_{\xi}:[0, \infty) \rightarrow D$ such that $\gamma_{\xi}(0)=b$ and $\lim _{t \rightarrow \infty} \gamma_{\xi}(t)=\xi$, if $D \subset \mathbb{C}$ is bounded strongly convex domain with $C^{3}$-boundary. We will denote the corresponding Busemann point by $h_{\xi}: D \rightarrow \mathbb{R}$, so

$$
h_{\xi}(z)=\lim _{t \rightarrow \infty} k_{D}\left(z, \gamma_{\xi}(t)\right)-k_{D}\left(b, \gamma_{\xi}(t)\right)
$$

Lemma 3.3 If $D \subset \mathbb{C}^{n}$ is a bounded strongly convex domain with $C^{3}$-boundary, then each horofunction of $\left(D, k_{D}\right)$ is a Busemann point and of the form $h_{\xi}$ for some $\xi \in \partial D$. Moreover, $\delta\left(h_{\xi}, h_{\eta}\right)=\infty$ if $\xi \neq \eta$. If $D=\prod_{i=1}^{r} D_{i}$, where each $D_{i}$ is a bounded strongly convex domain with $C^{3}$-boundary, then the horofunctions $h$ of $\left(D, k_{D}\right)$ are Busemann points and precisely the functions of the form,

$$
h(z)=\max _{j \in J} h_{\xi_{j}}\left(z_{j}\right)-\alpha_{j}
$$

where $J \subseteq\{1, \ldots, r\}$ non-empty, $\xi_{j} \in \partial D_{j}$ for $j \in J$, and $\min _{j \in J} \alpha_{j}=0$. 
Proof To prove the first assertion let $h \neq h^{\prime}$ be horofunctions of $\left(D, k_{D}\right)$. As $\left(D, k_{D}\right)$ is a proper geodesic metric space, we know there exist sequences $\left(w_{n}\right)$ and $\left(z_{n}\right)$ in $D$ such that $h_{w_{n}} \rightarrow h$ and $h_{z_{n}} \rightarrow h^{\prime}$. After taking subsequences, we may assume that $w_{n} \rightarrow \xi \in \partial D$ and $z_{n} \rightarrow \eta \in \partial D$, since $D$ has a compact norm closure and $h$ and $h^{\prime}$ are horofunctions.

We claim that $\xi \neq \eta$. To prove this, we need the assumption that $D \subset \mathbb{C}$ is a bounded strongly convex domain with $C^{3}$-boundary and use results by Abate [1] concerning the so-called small and large horospheres. These are defined as follows: for $R>0$, the small horosphere with centre $\zeta \in \partial D$ (and basepoint $b \in D$ ) is given by

$$
\mathcal{E}(\zeta, R):=\left\{x \in D: \limsup _{z \rightarrow \zeta} k_{D}(x, z)-k_{D}(b, z)<\frac{1}{2} \log R\right\}
$$

and the large horosphere with centre $\zeta \in \partial D$ (and basepoint $b \in D$ ) is given by

$$
\mathcal{F}(\zeta, R):=\left\{x \in D: \liminf _{z \rightarrow \zeta} k_{D}(x, z)-k_{D}(b, z)<\frac{1}{2} \log R\right\} .
$$

We note that the horoballs

$$
\mathcal{H}\left(h, \frac{1}{2} \log R\right)=\left\{x \in D: \lim _{n \rightarrow \infty} k_{D}\left(x, w_{n}\right)-k_{D}\left(b, w_{n}\right)<\frac{1}{2} \log R\right\}
$$

and

$$
\mathcal{H}\left(h^{\prime}, \frac{1}{2} \log R\right)=\left\{x \in D: \lim _{n \rightarrow \infty} k_{D}\left(x, z_{n}\right)-k_{D}\left(b, z_{n}\right)<\frac{1}{2} \log R\right\}
$$

satisfy

$$
\mathcal{E}(\xi, R) \subseteq \mathcal{H}\left(h, \frac{1}{2} \log R\right) \subseteq \mathcal{F}(\xi, R) \text { and } \mathcal{E}(\eta, R) \subseteq \mathcal{H}\left(h^{\prime}, \frac{1}{2} \log R\right) \subseteq \mathcal{F}(\eta, R)
$$

It follows from [2, Theorem 2.6.47] (see also [1]) that $\mathcal{E}(\xi, R)=\mathcal{H}\left(h, \frac{1}{2} \log R\right)=$ $\mathcal{F}(\xi, R)$ and $\mathcal{E}(\eta, R)=\mathcal{H}\left(h^{\prime}, \frac{1}{2} \log R\right)=\mathcal{F}(\eta, R)$, as $D$ is strongly convex and has $C^{3}$-boundary. Thus, if $\xi=\eta$, then $h=h^{\prime}$, since the horoballs, $\mathcal{H}(h, r)$ and $\mathcal{H}\left(h^{\prime}, r\right)$ for $r \in \mathbb{R}$, completely determine the horofunctions. This shows that $\xi \neq \eta$. It follows that each horofunction is of the form $h_{\xi}$, with $\xi \in \partial D$, and hence a Busemann point.

Now suppose that $h_{1}$ and $h_{2}$ are horofunctions, with $\left(z_{n}^{1}\right)$ converging to $h_{1}$ and $\left(z_{n}^{2}\right)$ converging to $h_{2}$. After taking a subsequence, we may assume that $z_{n}^{1} \rightarrow \xi_{1} \in \partial D$ and $z_{n}^{2} \rightarrow \xi_{2} \in \partial D$. We now show that if $\xi_{1} \neq \xi_{2}$, then $h_{1} \neq h_{2}$. This implies that there is a one-to-one correspondence between the horofunctions of $\left(D, k_{D}\right)$ and $\xi \in \partial D$.

To prove this, we note that as $D$ is strongly convex, $D$ is strictly convex, i.e. for each $v \neq \mu$ in $\partial D$, the open straight-line segment $(v, \mu) \subset D$. By [1, Theorem 1.7], we know that $\partial D \cap \operatorname{cl}\left(\mathcal{F}\left(h_{1}, R\right)\right)=\left\{\xi_{1}\right\}$ and $\partial D \cap \operatorname{cl}\left(\mathcal{F}\left(h_{2}, R\right)\right)=\left\{\xi_{2}\right\}$ for 
all $R>0$. This implies that $\partial D \cap \operatorname{cl}\left(\mathcal{H}\left(h_{1}, r\right)\right)=\left\{\xi_{1}\right\}$ and $\partial D \cap \operatorname{cl}\left(\mathcal{H}\left(h_{2}, r\right)\right)=$ $\left\{\xi_{2}\right\}$ for all $r \in \mathbb{R}$. Moreover, from [4, Lemma 5], we know that the straight-line segment $\left[b, \xi_{1}\right] \subset \operatorname{cl}\left(\mathcal{H}\left(h_{1}, 0\right)\right)$. There exists a neighbourhood $W \subset \mathbb{C}^{n}$ of $\xi_{1}$ such that $W \cap \operatorname{cl}\left(\mathcal{H}\left(h_{2}, 0\right)\right)=\emptyset$. If we let $w \in\left[b, \xi_{1}\right) \cap W$, then $h_{1}(w) \leq 0$, but $h_{2}(w)>0$, and hence $h_{1} \neq h_{2}$.

Now suppose that $\xi \neq \eta$ in $\partial D$. We know that $\partial D \cap \operatorname{cl}\left(\mathcal{H}\left(h_{\eta}, 0\right)\right)=\{\eta\}$ and $\gamma_{\xi}(t) \notin \operatorname{cl}\left(\mathcal{H}\left(h_{\eta}, 0\right)\right)$ for all $t>0$ large. So,

$$
H\left(h_{\xi}, h_{\eta}\right)=\lim _{t \rightarrow \infty} k_{D}\left(\gamma_{\xi}(t), b\right)+h_{\eta}\left(\gamma_{\xi}(t)\right) \geq \liminf _{t \rightarrow \infty} k_{D}\left(\gamma_{\xi}(t), b\right)=\infty,
$$

since $h_{\eta}\left(\gamma_{\xi}(t)\right) \geq 0$ for all $t$ large. This implies that $\delta\left(h_{\xi}, h_{\eta}\right)=\infty$.

The final part follows directly from Theorem 2.3 and Proposition 2.7.

The proof of Theorem 1.1 is now elementary.

Proof of Theorem 1.1 If $X_{j} \subset \mathbb{C}^{m_{j}}$ is a bounded convex domain, then $\left(X_{j}, k_{X_{j}}\right)$ is proper geodesic metric space which contains a geodesic ray by [2, Theorem 2.6.19]. Moreover, if $Y_{j} \subset \mathbb{C}^{n_{j}}$ is a bounded strongly convex domain with $C^{3}$-boundary, then by Lemma 3.3, all the horofunctions of $\left(Y_{j}, k_{Y_{j}}\right)$ are Busemann points and any two distinct Busemann points have infinite detour distance. So, Theorem 1.2 applies and gives the desired result.

Remark 3.4 I am grateful to Andrew Zimmer for sharing the following observations with me. In the case where $q=1$, Theorem 1.1 can be strengthened and shown in a variety of other ways. Indeed, it was shown by Balogh and Bonk [6] that the Kobayashi distance is Gromov hyperbolic on a strongly pseudo-convex domain with $C^{2}$-boundary, but the Kobayashi distance on a product domain is clearly not Gromov hyperbolic. This immediately implies Theorem 1.1 for $q=1$ in the more general case where the image domain is strongly pseudo-convex and has $C^{2}$-boundary.

In fact, if $q=1$ there exists a further strengthening of Theorem 1.1 which only requires the image domain to be strictly convex by using a local argument. The isometric embedding is a locally Lipschitz map with respect to the Euclidean norm, and hence differentiable almost everywhere by Rademacher's theorem. This implies that the embedding is also an isometric embedding under the Kobayashi infinitesimal metric. On strictly convex domains, the unit balls in the tangent spaces are strictly convex and in product domains, they are not, which yields a contradiction.

Finally, for holomorphic isometric embeddings and $q=1$, Theorem 1.1 can be extended to the case where the image domain is convex with $C^{1, \alpha}$-boundary, see [28, Theorem 2.22].

Looking at the conditions required in Theorem 1.2, it seems likely the regularity conditions on the domains $Y_{j}$ in Theorem 1.1 can be relaxed considerably. In particular, one may speculate that it suffices to assume that each domain $Y_{j}$ is strictly convex and has a $C^{1}$-boundary.

Acknowledgements The author wishes to thank the referee for their useful feedback and careful reading of the paper. 
Open Access This article is licensed under a Creative Commons Attribution 4.0 International License, which permits use, sharing, adaptation, distribution and reproduction in any medium or format, as long as you give appropriate credit to the original author(s) and the source, provide a link to the Creative Commons licence, and indicate if changes were made. The images or other third party material in this article are included in the article's Creative Commons licence, unless indicated otherwise in a credit line to the material. If material is not included in the article's Creative Commons licence and your intended use is not permitted by statutory regulation or exceeds the permitted use, you will need to obtain permission directly from the copyright holder. To view a copy of this licence, visit http://creativecommons.org/licenses/by/4.0/.

\section{References}

1. Abate, M.: Horospheres and iterates of holomorphic maps. Math. Z. 198, 225-238 (1988)

2. Abate, M.: Iteration Theory of Holomorphic Maps on Taut Manifolds. Research and Lecture Notes in Mathematics. Complex Analysis and Geometry. Mediterranean Press, Rende (1989)

3. Abate, M.: The Kobayashi distance in holomorphic dynamics and operator theory. In: Blanc-Centi, L. (ed.) Metrics and Dynamical Aspects in Complex Analysis. Springer, New York (2017)

4. Abate, M., Raissy, J.: Wolff-Denjoy theorems in nonsmooth convex domains. Ann. Mat. Pura Appl. (4) 193(5), 1503-1518 (2014)

5. Akian, M., Gaubert, S., Walsh, C.: The max-plus Martin boundary. Doc. Math. 14, 195-240 (2009)

6. Balogh, Z.M., Bonk, M.: Gromov hyperbolicity and the Kobayashi metric on strictly pseudoconvex domains. Comment. Math. Helv. 75(3), 504-533 (2000)

7. Bracci, F., Gaussier, H.: Horosphere topology. Ann. Sc. Norm. Super. Pisa Cl. Sci. (5) 20(1), 239-289 (2020)

8. Brouwer, L.E.J.: Beweis der Invarianz des $n$-dimensionalen Gebiets. Math. Ann. 71, 305-315 (1912)

9. Duchin, M., Fisher, N.: Stars at infinity in Teichmüller space. Geom. Dedicata 213, 531-545 (2021)

10. Gutiérrez, A.W.: The horofunction boundary of finite-dimensional $\ell_{p}$ spaces. Colloq. Math. 155(1), 51-65 (2019)

11. Kapeluszny, J., Kuczumow, T., Reich, S.: The Denjoy-Wolff theorem in the open unit ball of a strictly convex Banach space. Adv. Math. 143(1), 111-123 (1999)

12. Karlsson, A.: Elements of a metric spectral theory (2019). arXiv:1904.01398

13. Kobayashi, S.: Hyperbolic complex spaces. In: Grundlehren der Mathematischen Wissenschaften, vol. 318. Springer, Berlin (1998)

14. Lemmens, B., Nussbaum, R.: Nonlinear Perron-Frobenius Theory, Cambridge Tracts in Mathematics 189. Cambridge University, Press (2012)

15. Lemmens, B., Walsh, C.: Isometries of polyhedral Hilbert geometries. J. Topol. Anal. 3(2), 213-241 (2011)

16. Lemmens, B., Lins, B., Nussbaum, R.: Detecting fixed points of nonexpansive maps by illuminating the unit ball. Isr. J. Math. 224(1), 231-262 (2018)

17. Lemmens, B., Roelands, M., Wortel, M.: Isometries of infinite dimensional Hilbert geometries. J. Topol. Anal. 10(4), 941-959 (2018)

18. Lempert, L.: La métrique de Kobayashi et la représentation des domaines sur la boule. Bull. Soc. Math. France 109, 427-474 (1981)

19. Mahajan, P.: On isometries of the Kobayashi and Caratheodory metrics. Ann. Polon. Math. 104(2), 121-151 (2012)

20. Minsky, Y.N.: Extremal length estimates and product regions in Teichmüller space. Duke Math. J. 83(2), 249-286 (1996)

21. Papadopoulos, A.: Metric spaces, convexity and nonpositive curvature. In: IRMA Lectures in Mathematics and Theoretical Physics, 6. European Mathematical Society (EMS), Zürich (2005)

22. Poincaré, H.: Les fonctions analytiques de deux variables et la répresentation conforme. Rend. Circ. Mat. Palermo 23, 185-220 (1907)

23. Rieffel, M.A.: Group $C^{*}$-algebras as compact quantum metric spaces. Doc. Math. 7, 605-651 (2002)

24. Walsh, C.: The horofunction boundary of finite-dimensional normed spaces. Math. Proc. Camb. Philos. Soc. 142(3), 497-507 (2007)

25. Walsh, C.: The horofunction boundary of the Hilbert geometry. Adv. Geom. 8(4), 503-529 (2008) 
26. Walsh, C.: Gauge-reversing maps on cones, and Hilbert and Thompson isometries. Geom. Topol. 22(1), 55-104 (2018)

27. Walsh, C.: Hilbert and Thompson geometries isometric to infinite-dimensional Banach spaces. Ann. Inst. Fourier (Grenoble) 68(5), 1831-1877 (2018)

28. Zimmer, A.M.: Characterizing domains by the limit set of their automorphism group (2017). arXiv: 1506.07852

29. Zwonek, W.: Izometrie Zespolone, PhD Dissertation, Jagiellonian University, Kraków, (1994)

30. Zwonek, W.: The Carathéodory isometries between the products of balls. Arch. Math. (Basel) 65 , 434-443 (1995)

Publisher's Note Springer Nature remains neutral with regard to jurisdictional claims in published maps and institutional affiliations. 